\title{
Determination of Putrescine and Tyramine in Fish by Micellar Liquid Chromatography with UV Detection Using Direct Injection
}

\author{
Mei-Liang Chin-Chen ${ }^{1}$, Devasish Bose ${ }^{2}$, Josep Esteve-Romero ${ }^{1}$, Juan Peris-Vicente ${ }^{1}$, \\ Maria Rambla-Alegre ${ }^{1}$ and Samuel Carda-Broch ${ }^{*}, 1$
}

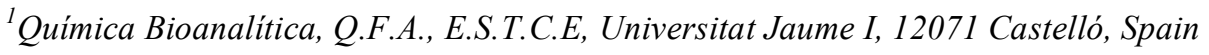

${ }^{2}$ Criminology and Forensic Sciences, Dr. H.S. Gour University, Sagar, India

\begin{abstract}
This paper shows how fish samples could be directly injected into a chromatograph, without any previous extraction, using micellar liquid chromatography, taking as an example the determination of two biogenic amines: putrescine and tyramine, derivatized with 3,5-dinitrobenzoyl chloride. First, the method has been optimized, using an interpretive model, and the best conditions for analysis were: mobile phase containing $0.15 \mathrm{M} \mathrm{SDS}-6 \%(v / v)$ butanol-pH 7, C18 column $(125 \times 0.46 \mathrm{~mm}, 5 \mu \mathrm{m}$ particle size $)$, UV detection of derivatives set at $260 \mathrm{~nm}$. Second, the method has been validated using spiked samples following the guidelines proposed by the US Food and Drug Administration: linear in the range from 0.5 to $1000 \mathrm{ppm}\left(\mathrm{r}^{2}>0.999\right)$, detection limits of 30 and $40 \mathrm{ppb}$ for putrescine and tyramine, respectively, a $500 \mathrm{ppb}$ limit of quantification in both cases, intra- and inter-day precision (RSD) below $2 \%$, and accuracy between $99.5 \%$ and $102.7 \%$. The method is adequate for routine analysis in the determination of these compounds in fish sauces.
\end{abstract}

Keywords: Micellar mobile phase, tyramine, putrescine, optimization, direct injection, fish.

\section{INTRODUCTION}

Putrescine and tyramine are biogenic amines present in foods either as natural products or after fermentation, decomposition or putrefaction processes [1]. Putrescine or 1,4-diaminobutane $\left(\mathrm{p} K_{a l}=9.71\right.$ and $\mathrm{p} K_{a 2}=11.15, \log P_{o / w}=$ -0.96) is largely responsible for the foul odour of putrefying flesh, but also contribute to the odour of such processes as bad breath and bacterial vaginosis. Tyramine or 4-hydroxyphenethylamine $\left(\mathrm{p} K_{a 1}=9.3\right.$ and $\left.\mathrm{p} K_{a 2}=10.4, \log P_{o / w}=0.98\right)$ [2] is a monoamine compound derived from the amino acid tyrosine. In foods, it is often produced by the decarboxylation of tyrosine during fermentation or decay. Foods containing considerable amounts of these biogenic amines include fish, alcoholic beverages, beef, chocolate, cheeses, pork and poultry.

Several analytical methods have been developed for the determination of biogenic amines. Most of these methods use high performance liquid chromatography (HPLC) with ultraviolet [3-9] or fluorescence [10] detection after precolumn derivatization with dansyl chloride [3], benzoyl chloride [4] and $o$-phthalaldehyde [10], or by using electrochemical detection [11]. Other methods are based on the capillary zone electrophoresis technique with conductometric [12] or pulsed amperometric detection [13], and also the micellar electrokinetic capillary chromatography method [14-18]. Other procedures, such as thin layer chromatography [19] have also been proposed. Recently, gas chromatography [20] and HPLC [21-23] separation,

*Address correspondence to this author at the Química Bioanalítica, Q.F.A., E.S.T.C.E, Universitat Jaume I, 12071 Castelló, Spain; Tel: +34964728095; Fax:+34964728066; E-mail: samuel.carda@qfa.uji.es followed by selective mass spectrometric detection, has become a method of choice but such selective, fast and sensitive methods are usually not suitable for routine clinical analysis due to financial reasons.

Nevertheless, direct application of a single chromatographic approach is not feasible for the simultaneous determination of biogenic amines for a number of reasons. First of all, the lack of a common chromophore or fluorophore in their structure usually makes it necessary to perform a derivatization step prior to detection, which involves introducing an easily detectable label group within the amine structure. The reagent 3,5-dinitrobenzoyl chloride (DNBZ-Cl) has been widely used as a chromophore to determine amines in food samples. Derivatization is quite fast (less than $5 \mathrm{~min}$ ), the reaction is quantitative and reproducible, and derivatives are stable and show high sensitivity [7]. Second, in almost all the approaches, derivatized amines have to undergo extraction in a suitable organic solvent, evaporation to dryness and re-dissolution, thereby introducing the risk of sample loss and contamination and increasing the analysis time. Finally, chromatographic conditions result in either insufficient separation or prolonged analysis, which may take longer than an hour to perform $[4,7,9]$.

Micellar liquid chromatography (MLC) allows complex matrices to be analysed without the aid of extraction and with direct injection of the samples. Micelles tend to bind proteins competitively, thereby releasing protein-bound drugs and proteins, rather than precipitating into the column. Proteins compounds are solubilized and washed harmlessly away, eluting with the solvent front. Micellar mobile phases usually need less quantity of organic modifier and generate less amount of toxic waste in comparison to aqueous-organic solvents, so that they are less toxic, non-flammable, 
biodegradable and relatively inexpensive [21]. MLC has proved to be a useful technique in the determination of diverse groups of compounds in several matrices [23-27], including food samples $[8,9,28,29]$.

The aim of this work was to develop a rapid, simple and selective procedure for determining putrescine and tyramine by means of a new MLC method using a C18 column and UV detection, prior derivatization with 3,5-dinitrobenzoyl chloride, and to demonstrate that the pretreatment of fish sauces can be dramatically reduced. The suggested methodology was validated in terms of linearity, sensitivity, LOD, LOQ, accuracy, precision and recovery, following the Food and Drug Administration guideline [30].

\section{EXPERIMENTAL}

\subsection{Apparatus and Instrumentation}

The chromatographic system was an Agilent Technologies Series 1100 (Palo Alto, CA, USA) equipped with a quaternary pump, a thermostated autosampler and a column compartment. Monitoring was performed after derivatization at $260 \mathrm{~nm}$ in the UV detector; the flow-rate and the injection volume were $1 \mathrm{~mL} / \mathrm{min}$, and $20 \mu \mathrm{L}$, respectively. A Kromasil C18 column ( $125 \mathrm{~mm} \times 4.6 \mathrm{~mm}, 5$ $\mu \mathrm{m}$ particle size) from Scharlab (Barcelona, Spain) was also used. The dead time was determined as the mean value of the first significant deviation from the baseline in the chromatograms. The signal was acquired by a PC computer connected to the chromatograph, through a HP Chemstation. The $\mathrm{pH}$ of the solutions was measured with a Crison GLP 22 (Barcelona) equipped with a combined $\mathrm{Ag} / \mathrm{AgCl} / \mathrm{glass}$ electrode. The balance used was a Mettler-Toledo AX-105 Delta-Range (Greifensee, Switzerland). The vortex shaker and sonification unit were from Selecta (Barcelona).

\subsection{Chemicals and Reagents}

The biogenic amines putrescine (Put) and tyramine (Tym) $(99.9 \%$ for both), and the reagent for derivatization (3,5-dinitrobenzoyl chloride) (98\% pure) were purchased from Sigma-Aldrich (St. Louis, MO, USA). The surfactant sodium dodecyl sulphate (99\% pure) was from Merck (Darmstadt, Germany); the organic solvents acetonitrile, propanol, butanol and pentanol were from Scharlab; sodium dihydrogen phosphate, hydrochloride acid and hydroxide sodium were from Panreac (Barcelona). All solutions were prepared in ultrapure water (Millipore, S.A.S. Molsheim, France). Biogenic amine solutions and fish sauces were filtered through $0.45 \mu \mathrm{m}$ nylon membranes (Millex-HN, Millipore, Bedford, MA 01730 USA). The corresponding hydrochlorides of putrescine and tyramine were dissolved in $0.1 \mathrm{M} \mathrm{HCl}$ to provide final concentrations of $100 \mathrm{~g} / \mathrm{mL}$.

\subsection{3,5-Dinitrobenzoyl Chloride Biogenic Amine Derivatization}

As a derivatizing reagent $5 \mathrm{mM}$ 3,5-dinitrobenzoyl chloride was dissolved in acetonitrile. Aliquots $(400 \mu \mathrm{L})$ of standards or sample, $1 \mathrm{M} \mathrm{NaOH}(1200 \mu \mathrm{L})$, 2-propanol (700 $\mu \mathrm{L})$ and 3,5-dinitrobenzoyl chloride $(2100 \mu \mathrm{L})$ were mixed in a reaction tube. After $3 \mathrm{~min}$ of shaking at $25^{\circ} \mathrm{C}, 2 \mathrm{M} \mathrm{HCl}$ $(1000 \mu \mathrm{L})$ was added to stop the reaction. Finally, after 1 min of shaking, derivatized biogenic amines were injected into the chromatographic system. Under these conditions, the formed derivatives are (DNBZ) 2 Put and (DNBZ)Tym [7].

\subsection{Chromatographic Conditions}

Derivatized biogenic amine separation was performed in a reversed-phase $\mathrm{C} 18$ Kromasil column thermostated at $25^{\circ} \mathrm{C}$. The mobile phase was an aqueous solution of $0.15 \mathrm{M}$ SDS and $0.01 \mathrm{M} \mathrm{NaH}_{2} \mathrm{PO}_{4}$, with $6 \%$ butanol (v/v) buffered at $\mathrm{pH}$ 7. The flow-rate, injection volume and UV wavelength were $1 \mathrm{~mL} / \mathrm{min}, 20 \mu \mathrm{L}$ and $260 \mathrm{~nm}$, respectively. Chromatographic signals were acquired and processed with an Agilent ChemStation (Rev. B.01.03).

\subsection{Sample Preparation}

Fish sauce samples of anchovies (Eugraulidea spp.) were obtained from a market. One portion of anchovies was stored without treatment and another was previously mixed with common salt in at a ratio of 75/25 (sample/salt w/w), a wellknown treatment to avoid food spoilage. Samples were stored in a fridge at $5^{\circ} \mathrm{C}$ for one month. Each sample was analyzed by three replicates. For each case herein considered, $1 \mathrm{~g}$ of each sample was mixed with $0.5 \mathrm{~mL}$ of ethanol and topped up to $10 \mathrm{~mL}$ with $0.1 \mathrm{M}$ SDS. In the case of spiking, the appropriate volume of analytes (solved in 0.1 $\mathrm{M} \mathrm{HCl}$ ) were mixed with $1 \mathrm{~g}$ of sample and stored one day to favor the contact between analytes and the sample, and solvent evaporation [31]. Then the spiked sample was mixed with $0.5 \mathrm{~mL}$ of ethanol and topped up to $10 \mathrm{~mL}$ with $0.1 \mathrm{M}$ SDS. An aliquot of the sample $(400 \mu \mathrm{L})$ was derivatized as explained in Section 2.3. Then samples were filtered and injected into the chromatograph. The three samples were treated and injected independently.

\section{RESULTS AND DISCUSSION}

\subsection{Optimization Strategy and Mobile Phase Selection}

First of all, SDS was selected as the surfactant because, apart from being the most widely used, it also reduces retention of compounds, has a high degree of sensitivity, and desorption and cleaning up processes are easy to perform. $\mathrm{pH}$ should remain constant to obtain reproducible retention times. Then the mobile phase was buffered at $\mathrm{pH} 7$, which allows an adequate separation of the 3,5-dinitrobenzoyl biogenic amine derivatives [7]. However, in a pure micellar mobile phase of SDS, the high retention time of putrescine and tyramine make it necessary to add a small amount of organic solvent in order to decrease the retention times and improve the efficiency.

Propanol, however, could not be used as an organic modifier for the separation of putrescine and tyramine, because, although the resolution was good, the retention time was long and this led to long analysis times. On the other hand, pentanol also overlap the two compounds. Then butanol was chosen as organic modifier.

In order to determine the optimal concentration of SDS and the amount of butanol, a chemometric tool was used, which considers the retention factor, efficiency and asymmetry. The accurate prediction of the retention behaviour, based on a proven model, can speed up the process of finding the optimal composition of the mobile phase for a given compound. The following equation has proved to be adequate for describing the retention of many 
compounds in MLC with hybrid mobile phases, with errors below $2 \%$ [32].

$$
k=\frac{K_{A S} \frac{1+K_{S D} \varphi}{1+K_{A D} \varphi}}{1+K_{A M} \frac{1+K_{M D} \varphi}{1+K_{A D} \varphi}[M]}
$$

where $k$ is the retention factor, [M] and $\varphi$ are the concentrations of surfactant and modifier, respectively; $\mathrm{K}_{\mathrm{AS}}$ and $\mathrm{K}_{\mathrm{AM}}$ describe the association equilibria between the solute in bulk water and the stationary phase or micelle, respectively; $\mathrm{K}_{\mathrm{SD}}, \mathrm{K}_{\mathrm{AD}}$ and $\mathrm{K}_{\mathrm{MD}}$ are constants that measure the relative variation in the concentration of solute in bulk water and micelles, due to the presence of modifier and referring to a pure micellar solution. In order to resolve the method, the behavior of the analytes in eight mobile phases must be considered. In order to determine the best mobile phase composition, biogenic amines were injected into mobile phases at $\mathrm{pH} 7$ containing the following amounts of SDS (M)/butanol $(\%, v / v): 0.15 / 4 ; 0.15 / 6 ; 0.125 / 4$; $0.125 / 3.5 ; 0.1 / 3 ; 0.1 / 4 ; 0.125 / 1 ; 0.125 / 5$. The data processing to obtain the optimal composition of the mobile phase was performed with the Michrom software [33]. After the modeling step and visualization of the different theoretical chromatograms, the optimum mobile phase was: $0.15 \mathrm{M}$ $\mathrm{SDS} / 6 \%(v / v)$ butanol-pH 7. Under these conditions, the retention times (in min) for biogenic amines were 3.8 for tyramine and 8.4 for putrescine. These times are significantly low if compared to those obtained by hydro-organic liquid chromatography using the same derivatizing reagent and detection at $260 \mathrm{~nm}(60 \mathrm{~min})$ [7]. The results of retention factor, efficiency and asymmetry are shown in Table $\mathbf{1 .}$

\subsection{Method Validation}

Using the optimum mobile phase, validation studies, under FDA guideline including the calibration parameters (slope, and intercept), selectivity, detection and quantification limits, intra- and inter-day precision and accuracy, and robustness studies (SDS and butanol concentrations, $\mathrm{pH}$ and flow rate) were performed. The overall validation was made using spiked samples of fresh anchovy paste.

Table 1. Retention Times $\left(\boldsymbol{R}_{t}\right)$, Retention Factors $(k)$, Efficiencies $(N)$ and Asymmetries $(B / A)$ of the Biogenic Amines Obtained Using the 0.15 M SDS6\% (v/v) Butanol-pH 7 Mobile Phase

\begin{tabular}{|c|c|c|c|c|}
\hline Biogenic Amine & $\boldsymbol{R}_{\boldsymbol{t}}$ & $\boldsymbol{k}$ & $\boldsymbol{N}$ & $\boldsymbol{B} / \boldsymbol{A}$ \\
\hline \hline Putrescine & 3.8 & 3.16 & 5000 & 1.014 \\
\hline Tyramine & 8.4 & 8.19 & 6200 & 1.025 \\
\hline
\end{tabular}

The selectivity of the method was tested by spiking a sample of blank fresh anchovy paste and another spiked with $100 \mathrm{ppm}$ of putrescine and $200 \mathrm{ppm}$ of tyramine. As seen in Fig. (1a). near the retention time corresponding to the analytes, no other peaks were detected. The Fig. (1b) shows that putrescine and tyramine appear separated without overlapping with other compounds. The excess of reagent elutes at the dead time and do not provoke interferences. The derivatization reaction is not influenced by the fish sauce media as the medium is later fixed by adding SDS solution, organic solvents and sodium hydroxide and by diluting the original medium (see Sections 2.3 and 2.5). Other amines in the matrix would also react with DNBZ-Cl and could appear in the chromatogram. However, no other compounds were observed. It is important to consider that proteins were not hydrolyzed, so the amount of interfering amino acids would be very low.

Calibration curves were constructed using the areas of the chromatographic peaks obtained at seven different concentrations, each one by triplicate, in the $0.5-1000 \mathrm{ppm}$ range for putrescine and tyramine. To study the variability of the calibration parameter, the curves were obtained for 5 days over a period of two months for a different set of standards. The slope and intercept were determined by the method of least square linear regression analysis. Coefficients of


Fig. (1). Chromatogram of fresh anchovy paste analyzed following the proposed method, after derivatization with 3,5-dinitrobenzyl chloride: a) blank, and b) putrescine (100 ppm) and tyramine (200 ppm). Peaks are: (1) tyramine and (2) putrescine. 
Table 2. Inter-Day and Intra-Day Precision and Accuracy

\begin{tabular}{|c|c|c|c|c|c|c|c|}
\hline Compound & $\begin{array}{l}\text { Added Concentration } \\
\qquad(\mu \mathrm{g} / \mathrm{ml})\end{array}$ & $\begin{array}{c}\text { Found }^{\mathrm{a}} \\
(\text { Mean } \pm \text { SD })(\mu \mathrm{g} / \mathrm{ml})\end{array}$ & $\begin{array}{l}\text { Accuracy } \\
(\%)\end{array}$ & $\begin{array}{c}\text { Intra-Day C.V. } \\
(\%)\end{array}$ & $\begin{array}{c}\text { Found }^{\mathrm{b}}(\text { Mean } \pm \text { SD }) \\
(\mu \mathrm{g} / \mathrm{ml})\end{array}$ & $\begin{array}{l}\text { Accuracy } \\
(\%)\end{array}$ & $\begin{array}{c}\text { Inter-Day C.V. } \\
(\%)\end{array}$ \\
\hline \multirow{3}{*}{ Putrescine } & 0.2 & $0.205 \pm 0.003$ & +1.5 & 1.5 & $0.205 \pm 0.004$ & +2.5 & 2.0 \\
\hline & 1.0 & $1.013 \pm 0.018$ & +1.3 & 1.8 & $1.027 \pm 0.02$ & +2.7 & 1.9 \\
\hline & 10 & $9.95 \pm 0.09$ & -0.5 & 0.9 & $10.15 \pm 0.18$ & +1.5 & 1.8 \\
\hline \multirow{3}{*}{ Tyramine } & 0.2 & $0.202 \pm 0.004$ & +1.0 & 2.0 & $0.198 \pm 0.004$ & -1.0 & 2.0 \\
\hline & 1.0 & $0.996 \pm 0.017$ & -0.4 & 1.7 & $1.02 \pm 0.02$ & +2.0 & 2.0 \\
\hline & 10 & $10.03 \pm 0.13$ & +0.3 & 1.3 & $9.92 \pm 0.16$ & -0.8 & 1.6 \\
\hline
\end{tabular}

${ }^{a} \mathrm{n}=10,{ }^{b} \mathrm{n}=10$

determination $\left(r^{2}\right)$ were always higher than 0.9999 . Slope and intercept were $0.60 \pm 0.01$ and $0.02 \pm 0.01$, and $0.68 \pm 0.01$ and $0.03 \pm 0.01$, for putrescine and tyramine, respectively. Limits of detection (LOD, 3s criterion) for derivatized biogenic amines using the proposed method $(\mathrm{n}=10)$ were 30 and $40 \mathrm{ppb}$, for putrescine and tyramine, respectively. The LOQ was set as the minimum concentration reached by the calibration curve, 500 $\mathrm{ppb}$ in both cases. The regression coefficient was higher and the LOD was significantly lower than that found by hydro-organic liquid chromatography (LOD $124 \mathrm{ppb)} \mathrm{[7].}$

The intra- and inter-day precision and accuracy of putrescine and tyramine quantifications were determined by an analysis of spiked samples in a micellar solution at three different concentrations: $0.2,1$ and $10 \mathrm{ppm}$. The intra-day values were determined by assaying these three test solutions ten times $(n=10)$ on the same day, and inter-day values was the average of ten measurements of intra-day values taken on ten days $(n=10)$ over a 3 -month period. These measurements were made by different analysts and using different equipment at the same three biogenic amines concentrations. The results are shown in Table 2. Precision, which was defined as the percentage of relative standard deviation (RSD, \%) for intraand inter-day values, were below $2 \%$ in all cases. Accuracy for the intra- and interday values, taken as the quotient between the measured and know concentrations, was between $99.5 \%$ and $102.7 \%$. The high accuracy and precision indicate that the derivatization reaction is reproducible end quantitative.

Robustness of the method was examined by replicate injections $(\mathrm{n}=6)$ of standard solution at a concentration of 10 ppm under small changes in the chromatographic parameters
(SDS and butanol concentrations, $\mathrm{pH}$ and flow rate). Insignificant differences in peak areas and less variability in retention time were observed (see Table $\mathbf{3}$ ). Results indicate that the selected factors remain unaffected by small variations in these parameters: RSD was below $1.5 \%$.

\subsection{Analysis of Food Samples}

Fermented fish sauces of anchovies (Engraulidea spp.), produced by the breakdown of small fish compounds by enzymes, were analyzed. Samples $(n=10)$ were anchovy sauce, both without treatment and treated with table salt at the 75:25 $(w / w)$ ratio. Samples were prepared and stored in a fridge $\left(5^{\circ} \mathrm{C}\right)$ for a 30-day period, and were then analyzed. In the non-treated sample, the putrescine and tyramine concentrations were 235 and $625 \mathrm{ppm}$, respectively. For the salted sample, the amounts of putrescine and tyramine were $2 \mathrm{ppm}$ and $0.5 \mathrm{ppm}$, respectively. In the first case, the high amount of biogenic amines indicates spoilage, but a $25 \%$ treatment can prevent the fish sauce from degradation.

\section{CONCLUSIONS}

The results prove that micellar liquid chromatography procedure can be used for the analysis of putrescine and tyramine in less than $10 \mathrm{~min}$. The reagent 3,5-dinitrobenzoyl chloride was found to be highly suitable to analyze biogenic amines: the reaction is carried out quickly at room temperature by following a simple experimental procedure, the reaction is quantitative and reproducible, and the derivative is quite stable and provides high sensitivity. The analytical method is simple, rapid, effective and does not require a previous extraction step: the derivatized sample is directly injected into the

Table 3. Evaluation of the Robustness of the MLC Method

\begin{tabular}{|c|c|c|c|c|}
\hline Biogenic Amine & Changes in the Mobile Phase Parameters & Level & Retention Time (min) (RSD, \%) & Area (Arbitrary Unit) (RSD, \%) \\
\hline \hline \multirow{3}{*}{ Putrescine } & Concentration SDS (M) & $0.145-0.155$ & $3.82 \pm 0.05(1.3)$ & $6.10 \pm 0.09(1.5)$ \\
\cline { 2 - 5 } & $\mathrm{pH}$ & $6.9-7.1$ & $3.78 \pm 0.03(0.8)$ & $5.98 \pm 0.05(0.9)$ \\
\cline { 2 - 5 } & Flow (mL/min) & $0.98-1.02$ & $3.79 \pm 0.4(1.1)$ & $6.00 \pm 0.08(1.3)$ \\
\cline { 2 - 5 } & Buthanol (\%) & $5.9-6.1$ & $3.81 \pm 0.04(1.0)$ & $6.02 \pm 0.07(1.2)$ \\
\hline \multirow{3}{*}{ Tyramine } & Concentration SDS (M) & $0.145-0.155$ & $8.42 \pm 0.08(1.0)$ & $6.88 \pm 0.08(1.1)$ \\
\cline { 2 - 5 } & $\mathrm{pH}$ & $6.9-7.1$ & $8.38 \pm 0.03(0.4)$ & $6.84 \pm 0.03(0.4)$ \\
\cline { 2 - 5 } & Flow (mL/min) & $0.98-1.02$ & $8.44 \pm 0.13(1.5)$ & $6.79 \pm 0.06(0.9)$ \\
\cline { 2 - 5 } & Buthanol (\%) & $5.9-6.1$ & $8.40 \pm 0.11(1.3)$ & $6.82 \pm 0.04(0.6)$ \\
\hline
\end{tabular}


chromatographic system. Similar attempts can be made for the differentiation of other biologically attractive substances. The suggested methodology was successfully applied to study the degree of spoilage of anchovy sauce via the amount of biogenic amines. It was proved that a previous treatment with salt improves storage. The method is adequate for use in pollution surveys quality control and routine analyses.

\section{ACKNOWLEDGEMENTS}

This work was supported by the projects from Fundació Caixa Castelló-Bancaixa P1-1B2006-12 and Spanish Ministry of Education and Science (MEC) CTQ 200764473/BQU. MeiLiang Chin-Chen and Devasish Bose also thanks the Foundation for her grant. Maria Rambla-Alegre also wishes to thank the MEC for her FPU grant.

\section{REFERENCES}

[1] Izquierdo-Pulido, M.; Hernandez-Jover, T.; Mariné-Font, A.; VidalCarou, M.C. Biogenic Amines in European Beers. J. Agric. Food Chem. 1996, 44, 3159-3163.

[2] Moffat, A.C.; Osselton, M.D.; Brian, W. Clarke's Analysis of Drugs and Poisons. Pharmaceutical Press, London UK, 2004.

[3] Soufleros, E.H.; Bouloumpasi, E.; Zotou A.; Loukou Z. Determination of biogenic amines in Greek wines by HPLC and ultraviolet detection after dansylation and examination of factors affecting their presence and concentration Food Chem., 2007, 101, 704-716.

[4] Paleologos, E.K.; Chytiri, S.D.; Savvaidis, I.N.; Kontominas M.G. Determination of biogenic amines as their benzoyl derivatives after cloud point extraction with micellar liquid chromatographic separation J. Chromatogr. A, 2003, 1010, 217-224.

[5] Wood, A.T.; Hall, M.R. Reversed-phase high-performance liquid chromatography of catecholamines and indoleamines using a simple gradient solvent system and native fluorescence detection. $J$. Chromatogr. B, 2000, 744, 221-225.

[6] Moret, S.; Conte, L.S. High-performance liquid chromatographic evaluation of biogenic amines in foods an analysis of different methods of sample preparation in relation to food characteristics. J. Chromatogr. A, 1996, 729, 363-369.

[7] Kirschbaum, J.; Rebscher, K.; Bruckner, H. Liquid chromatographic determination of biogenic amines in fermented foods after derivatization with 3,5-dinitrobenzoyl chloride. J. Chromatogr. A 2000, 881, 517-530.

[8] Gil-Agustí, M.; Esteve-Romero, J.; Carda-Broch, S. Anserine and carnosine determination in meat samples by pure micellar liquid chromatography. J. Chromatogr. A, 2008, 1189, 444-450.

[9] Raviolo, M.A.; Rambla-Alegre, M.; Clausell-Tormos, J.; EsteveRomero, J.; Carda-Broch, S.; Capella-Peiró, M.E. Determination of sulfonamides in milk after precolumn derivatisation by micellar liquid chromatography. Anal. Chim. Acta 2007, 593, 152-156.

[10] Busto, O.; Miracle, M.; Guasch, J.; Borrull, F. Determination of biogenic amines in wines by high-performance liquid chromatography with on-column fluorescence derivatization. J. Chromatogr. A, 1997, 757, 311-318.

[11] Auger, J. ; Boulay, R.; Jaillais, B. ; Delion-Vancassel, S. Analysis of biogenic amines by solid-phase microextraction and high-performance liquid chromatography with electrochemical detection. J. Chromatogr. A, 2000, 870, 395-403.

[12] Kvasnička, F.; Voldřich, M. Determination of biogenic amines by capillary zone electrophoresis with conductometric detection. $J$. Chromatogr. A, 2006, 1103, 145-149.

[13] Sun, X.; Yang, X.; Wang, E. Determination of biogenic amines by capillary electrophoresis with pulsed amperometric detection. $J$. Chromatogr. A, 2003, 1005, 189-195.

[14] Su, S.C.; Chou, S.S.; Chang, P.C.; Hwang, D.F. Determination of biogenic amines in fish implicated in food poisoning by micellar electrokinetic capillary chromatography. J. Chromatogr. B, 2000, 749, 163-169.

[15] Liu, Y.M.; Cheng, J.K. Separation of biogenic amines by micellar electrokinetic chromatography with on-line chemiluminescence detection. J. Chromatogr. A 2003, 1003, 211-216.

[16] Butehorn, Ü.; Pyell, U. Simultaneous use of urea and acetonitrile as organic modifiers for optimization of resolution in micellar electrokinetic chromatography. J. Chromatogr. A 1997, 792157.

[17] Dorsey, J.G. Micellar liquid chromatography. Adv. Chromatogr. 1987, 27, 167-214.

[18] Berthod, A.; Dorsey, J.G. n-Octanol-water partition coefficient estimation by micellar electrokinetic capillary chromatography. Anal. Chem., 1995, 67, 744-749.

[19] Shalaby, A.R. Separation, identification and estimation of biogenic amines in foods by thin-layer chromatography. Food Chem. 1994, 59, 305-310.

[20] Yamamoto, S.; Itano, H.; Kataoka, H.; Makita, M.; Gas-liquid chromatographic method for analysis of $\mathrm{Di}$ - and polyamines in foods. $J$. Agric. Food Chem. 1982, 30, 435-439.

[21] Berthod, A.; García-Alvarez-Coque, M.C. Micellar liquid chromatography, Chromatographic Science Series, vol. 83, Marcel Dekker, New York, 2000.

[22] Berthod, A.; Girard, I.; Gonnet, C. Micellar liquid chromatography. Retention study of solutes of various polarities. Anal. Chem. 1986, 58, 1359-1362.

[23] Carda-Broch, S.; Esteve-Romero, J.S.; García-Alvarez-Coque, M.C. Liquid chromatographic determination of some thiazide diuretics in pharmaceuticals with a sodium dodecyl sulfate mobile phase. Analyst 1998, 123, 301-306.

[24] Esteve-Romero, J.; Ochoa-Aranda, E.; Bose, D.; Rambla-Alegre, M.; Peris-Vicente, J.; Martinavarro-Domínguez, A. Tamoxifen monitoring studies in breast cancer patients by micellar liquid chromatography Anal. Bioanal. Chem., 2010, 397, 1557-1561.

[25] Torres-Cartas, S.; Villanueva-Camañas, R.M.; García-Alvarez-Coque, M.C. Retention-structure relationship studies for some steroidal hormones in micellar liquid chromatography. Chromatographia 2000, 51, 577-585.

[26] Ruiz-Angel, M.J.; Carda-Broch, S.; Torres-Lapasió, J.R.; SimóAlfonso, E.F.; García-Alvarez-Coque, M.C. Micellar-organic versus aqueous-organic mobile phases for the screening of B-blockers. Anal. Chim. Acta 2002, 45, 109-123.

[27] Gil-Agustí, M; Carda-Broch, S.; García-Alvarez-Coque, M.C.; EsteveRomero, J. Micellar liquid chromatography determination of anticonvulsant drugs in pills and capsules. J. Liq. Chromatogr. Relat. Technol. 2000, 23, 1387-1401.

[28] Rambla-Alegre, M.; Peris-Vicente, J.; Marco-Peiró, S.; BeltránMartinavarro, B.; Esteve-Romero, J. Development of an analytical methodology to quantify melamine in milk using micellar liquid chromatography and validation according to EU Regulation 2002/654/EC. Talanta 2010, 81, 894-900.

[29] Rambla-Alegre, M.; Peris-Vicente, J.; Esteve-Romero, J.; Carda-Broch, $\mathrm{S}$. Analysis of selected veterinary antibiotics in fish by micellar liquid chromatography with fluorescence detection and validation in accordance with regulation 2002/657/EC. Food Chem. 2010, 123, 1294-1302.

[30] Guidance for Industry. Bioanalytical Method Validation. U.S Department of Health and Human Services. Food and Drug Administration, Rockville, MD, USA. 2001.

[31] Cano-Sancho, G.; Marin, S.; Ramos, A.J.; Peris-Vicente, J.; Sanchis, V; Occurrence of aflatoxin M1 and exposure assessment in Catalonia (Spain). Revista Iberoamericana de Micología 2010, 27, 130-135.

[32] García-Alvarez-Coque, M.C.; Torres-Lapasió, J.R.; Baeza-Baeza, J.J. Modelling of retention behaviour of solutes in micellar liquid chromatography. J. Chromatogr. A 1997, 780, 129-148.

[33] Torres-Lapasió, J.R. Michrom Software, eds. Micellar liquid chromatography, appendix. Marcel-Dekker, New York, USA, 2000, 501 . 